\title{
Preserving brightness in histogram equalization based contrast enhancement techniques
}

\begin{abstract}
Histogram equalization (HE) has been a simple yet effective image enhancement technique. However, it tends to change the brightness of an image significantly, causing annoying artifacts and unnatural contrast enhancement. Brightness preserving bi-histogram equalization (BBHE) and dualistic sub-image histogram equalization (DSIHE) have been proposed to overcome these problems but they may still fail under certain conditions. This paper proposes a novel extension of BBHE referred to as minimum mean brightness error bihistogram equalization (MMBEBHE). MMBEBHE has the feature of minimizing the difference between input and output image's mean. Simulation results showed that MMBEBHE can preserve brightness better than BBHE and DSIHE. Furthermore, this paper also formulated an efficient, integer-based implementation of MMBEBHE. Nevertheless, MMBEBHE also has its limitation. Hence, this paper further proposes a generalization of BBHE referred to as recursive mean-separate histogram equalization (RMSHE). RMSHE is featured with scalable brightness preservation. Simulation results showed that RMSHE is the best compared to HE, BBHE, DSIHE, and MMBEBHE.
\end{abstract}

Keyword: Bi-histogram equalization; Dualistic sub-image; Histogram equalization; Mean separate; Minimum mean brightness error; Recursive 